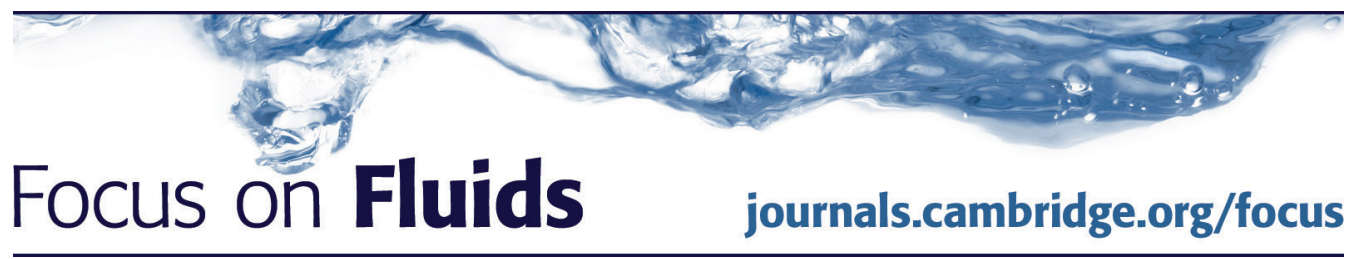

\title{
Polymer turbulence with Reynolds and Riemann
}

\author{
Michael D. Graham ${ }^{1,2, \dagger}$ \\ ${ }^{1}$ Department of Chemical and Biological Engineering, \\ University of Wisconsin-Madison, Madison, WI 53706, USA \\ ${ }^{2}$ Kavli Institute for Theoretical Physics, University of \\ California, Santa Barbara, CA 93106, USA
}

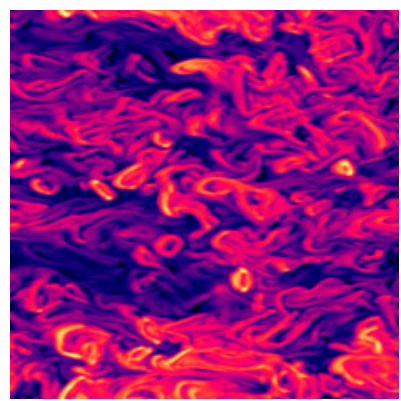

Models of flowing complex fluids such as polymer solutions often use a conformation tensor that reflects the state of the fluid microstructure. In polymer solutions, this quantity measures the orientation and stretching of the molecules, and reflects the fact that the squared length of a polymer molecule must be positive. By exploiting results from differential geometry and continuum mechanics, Hameduddin et al. (J. Fluid Mech., vol. 842, 2018, pp. 395-427) introduce a new approach for analysing the conformation tensor that respects this positivity constraint. With this approach, they present computational results for turbulent flow of a polymer solution that exhibits turbulent drag reduction, showing that the new measures of polymer stretching afforded by their approach lend insights not available in traditional methods.

Key words: drag reduction, non-Newtonian flows, turbulent flows

\section{Introduction}

Flows involving complex or multiphase fluids such as polymer or surfactant solutions, particulate suspensions or droplet emulsions arise in a wide range of natural and technological processes. For example, polymer or surfactant additives in a fluid can lead to substantial reduction of drag and energy consumption in turbulent flows. Recent work has introduced a number of new approaches, concepts and observations that are improving our understanding of this phenomenon (Doering, Eckhardt \& Schumacher 2006; Samanta et al. 2013; Graham 2014; Wang et al. 2014). More broadly, linear and nonlinear instabilities arise in flows of many complex fluids, leading to strongly time-dependent flows even when inertial effects are negligible (Shaqfeh 1996; Arratia et al. 2006; Pan et al. 2013). The paper we highlight here (Hameduddin et al. 2018) describes a novel approach that incorporates ideas from continuum mechanics and differential geometry to develop new measures of the distributions of polymer stretching in time-dependent flow of a polymer solution.

$\dagger$ Email address for correspondence: mdgraham@wisc.edu 
In simulating flows of complex fluids such as polymer solutions, we often use a simplified model of the fluid microstructure that results in an evolution equation for a second-order conformation tensor $\boldsymbol{C}$, which reflects the stretching and orientation of the polymer molecules. Because the squared length of any polymer molecules in the flow must be positive, $\boldsymbol{C}$ must be positive definite; i.e., $\boldsymbol{w} \cdot \boldsymbol{C} \cdot \boldsymbol{w}>0$ for any non-zero vector $\boldsymbol{w}$. This property is often indicated by simply writing ' $\boldsymbol{C}>0$ '.

The Reynolds decomposition $\boldsymbol{v}(\boldsymbol{x}, t)=\overline{\boldsymbol{v}}(\boldsymbol{x})+\boldsymbol{v}^{\prime}(\boldsymbol{x}, t)$, where $\boldsymbol{v}$ is a velocity field that depends on position $\boldsymbol{x}$ and time $t$, and $\vec{r}$ is a time average, is central to the analysis of turbulent and other time-dependent flows. For a time-dependent flow, we can of course write the Reynolds decomposition for $\boldsymbol{C}$ as well, $\boldsymbol{C}(\boldsymbol{x}, t)=\overline{\boldsymbol{C}}(\boldsymbol{x})+\boldsymbol{C}^{\prime}(\boldsymbol{x}, t)$, and we can imagine analysing $\boldsymbol{C}^{\prime}$ just as is done with $\boldsymbol{v}^{\prime}$. There is an important difference between the quantities, however. Whereas the velocity can take on any vector value, the conformation tensor $\boldsymbol{C}$ can only take on values such that $\boldsymbol{C}>0$. This physical constraint is at the heart of the work of Hameduddin et al. (2018).

\section{Overview}

To understand how to incorporate the positivity constraint into an analysis of $\boldsymbol{C}$, we consider first the simpler situation of a scalar real variable $x$ that is restricted to be positive, $x \in \mathbb{R}_{>0}$. In the present case, we could think of the squared length of a polymer molecule. Let us think about moving around in $\mathbb{R}_{>0}$ according to the differential equation

$$
\frac{\mathrm{d} s}{\mathrm{~d} t}=c,
$$

where $s(t)$ is the arclength along the half-line $x>0$ and $c$ is a real constant. With this equation, the arclength is just linear in time, $s(t)=c t$. So far, we have not specified the relation between an infinitesimal increment of arclength $\mathrm{d} s$ and an infinitesimal increment $\mathrm{d} x$. If we use the Euclidean relationship $\mathrm{d} s=\mathrm{d} x$, then, with initial condition $x=x(0)>0,(2.1)$ integrates to $s(t)=x(t)-x(0)=c t$. Clearly, if $c<0$, then for large enough time, $x(t)$ will become negative, i.e. it will leave the set that it is supposed to be restricted to.

Now, imagine that instead of using the Euclidean expression $\mathrm{d} s=\mathrm{d} x$, we measure arclength with the formula

$$
\mathrm{d} s=\frac{1}{x} \mathrm{~d} x
$$

Since it is position-dependent, this expression is an example of a non-Euclidean, and specifically a Riemannian arclength formula. It measures the relative distance between $x$ and $x+\mathrm{d} x$. Now, (2.1) integrates to

$$
s(t)=\log \frac{x(t)}{x(0)}=c t .
$$

Thus, $x(t)=x(0) \exp (c t)>0$ for all $x(0)>0$ and any $c$ : if we move according to this arclength formula, we will never leave the set $\mathbb{R}_{>0}$. Intuitively, we can understand this by observing that, with distance metric (2.2), $\mathrm{d} s / \mathrm{d} x$ diverges as $x$ decreases: zero is infinitely far from any positive number. Finally, for any two positive numbers $p$ and $q$, we can always set $x(0)=p$ and find $c$ and $t$ such that $x(t)=q$. The distance $d_{R}(p, q)$ between these points is $|s(t)|$, which from (2.3) is simply

$$
d_{R}(p, q)=\left|\log \frac{q}{p}\right|=|\log q-\log p| .
$$


(a)

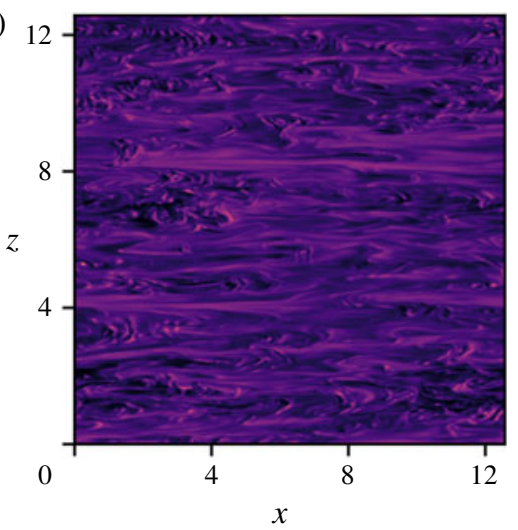

(b)

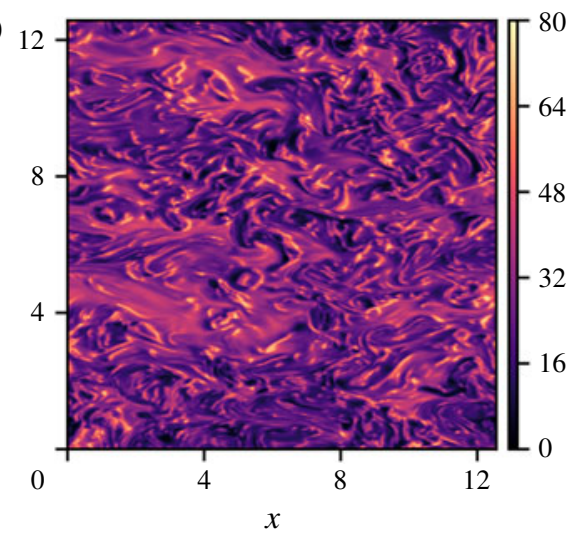

FIGURE 1. Contours of Riemannian distance $\kappa$ between instantaneous and mean conformation tensors at planes of constant wall-normal position $y^{+}$in viscoelastic channel flow: $(a) y^{+}=15$ (near the bottom wall) and $(b) y^{+}=180$ (at the centreline). Reproduced with permission from Hameduddin et al. (2018, figure 6(c,d)).

Logarithmic intensity and distance formulae are widely used, from the decibel scale for sound, to the Richter scale for earthquake intensity, to the $\mathrm{pH}$ scale for hydrogen ion concentration in aqueous solutions.

For positive definite matrices, closely analogous results have been developed (Lang 1999). For $\boldsymbol{X}>0$, (2.2) generalizes to

$$
\mathrm{d} s=\left(\operatorname{Tr}\left(\boldsymbol{X}^{-1} \mathrm{~d} \boldsymbol{X}\right)^{2}\right)^{1 / 2}
$$

and the Riemannian distance between positive definite matrices $\boldsymbol{P}$ and $\boldsymbol{Q}$ is given by a relation analogous to (2.4),

$$
d_{R}(\boldsymbol{P}, \boldsymbol{Q})=\left\|\log \left(\boldsymbol{P}^{-1} \cdot \boldsymbol{Q}\right)\right\|_{F},
$$

where $\|\boldsymbol{X}\|_{F}=\left(\operatorname{Tr}\left(\boldsymbol{X}^{\mathrm{T}} \cdot \boldsymbol{X}\right)\right)^{1 / 2}$ is the Frobenius norm of $\boldsymbol{X}$.

Hameduddin et al. (2018) apply the distance formula, (2.6), and some related results to the conformation tensor in viscoelastic flow. The squared Riemannian distance $\kappa$ between the instantaneous conformation $\boldsymbol{C}(t)$ and the mean conformation $\overline{\boldsymbol{C}}$ is simply

$$
\kappa=d_{R}^{2}(\overline{\boldsymbol{C}}, \boldsymbol{C}(t))=\left\|\log \left(\overline{\boldsymbol{C}}^{-1} \cdot \boldsymbol{C}(t)\right)\right\|_{F}^{2} .
$$

The conformation tensor at any instant can be transformed to $\boldsymbol{G}(t)=\overline{\boldsymbol{C}}^{-1 / 2} \cdot \boldsymbol{C}(t) \cdot \overline{\boldsymbol{C}}^{-1 / 2}$. Under this transformation, $\overrightarrow{\boldsymbol{C}}$ becomes the identity $\boldsymbol{I}$ and (2.7) can be rewritten $\kappa=d_{R}^{2}(\boldsymbol{I}, \boldsymbol{G})$. A measure of anisotropy of the instantaneous configuration relative to the mean can also be defined, $\xi=\inf _{a} d_{R}^{2}(a \overline{\boldsymbol{C}}, \boldsymbol{C}(t))=\inf _{a} d_{R}^{2}(a \boldsymbol{I}, \boldsymbol{G}(t))$. That is, $\xi$ is the distance between $\boldsymbol{G}$ and the nearest isotropic tensor. Finally, based on the geometric interpretation of the product of the eigenvalues of a tensor as a measure of its 'volume', Hameduddin et al. (2018) define a logarithmic volume ratio $\zeta=\log \operatorname{det} \boldsymbol{C}-\log \operatorname{det} \overline{\boldsymbol{C}}$.

To illustrate the usefulness of these new measures of polymer conformation, Hameduddin et al. (2018) present results from direct numerical simulations of flow of a polymer solution in a plane channel under conditions where the polymer leads to $60 \%$ drag reduction relative to the Newtonian flow. Figure 1 shows instantaneous contours of the distance measure $\kappa(2.7)$ at two wall-normal positions. A number 
of important features of the flow become apparent in this representation. First, the value of $\kappa$ is generally much larger at the centreline than near the wall. Recalling that this Riemannian distance is a relative distance measure, we see immediately that the relative magnitude of the fluctuations near the centre is much larger than near the wall. A plot of the analogous results for $\left\|\boldsymbol{C}^{\prime}\right\|_{F}$ would not reveal this information. We also see that regions of constant $\kappa$ are much more elongated in the flow $(x)$ direction near the wall than at the centre, revealing important differences in the anisotropy of the spatial structure of the flow. Another interesting result that would not arise from the usual Euclidean analysis is that the anisotropy $\xi$ of the conformation tensor relative to the mean displays, to a very good approximation, a logarithmic variation with distance from the wall. Logarithmic scalings in wall turbulence may indicate self-similarity, so this observation is worthy of further examination.

\section{Future}

The Riemannian quantities introduced by Hameduddin et al. (2018) provide a new view of polymer dynamics during turbulent flow. Strongly time-dependent flows of complex fluids arise in many other contexts as well, and the new approach described here may also lend insights into these flows. Many other complex fluids including droplet suspensions (Barthes-Biesel \& Rallison 1981) and blood (Moyers-Gonzalez, Owens \& Fang 2008) are also treated with conformation tensor models, so these are also natural candidates for analysis with the approach described here. Even in the context of Newtonian flow, positive definite tensors appear in the form of the convective momentum flux, $\rho \boldsymbol{v} v$. Subgrid-scale modelling of this quantity is of central interest in large-eddy simulations of turbulent flows (Meneveau \& Katz 2000). Perhaps the geometric decomposition introduced here can lead to new ways to make simplified models for complex flows.

\section{References}

Arratia, P. E., Thomas, C. C., Diorio, J. \& Gollub, J. P. 2006 Elastic instabilities of polymer solutions in cross-channel flow. Phys. Rev. Lett. 96, 144502.

BARthes-Biesel, D. \& Rallison, J. M. 1981 The time-dependent deformation of a capsule freely suspended in a linear shear-flow. J. Fluid Mech. 113, 251-267.

Doering, C., Eckhardt, B. \& Schumacher, J. 2006 Failure of energy stability in Oldroyd-B fluids at arbitrarily low Reynolds numbers. J. Non-Newtonian Fluid Mech. 135 (2-3), 92-96.

Graham, M. D. 2014 Drag reduction and the dynamics of turbulence in simple and complex fluids. Phys. Fluids 26, 101301.

Hameduddin, I., Meneveau, C., Zaki, T. A. \& Gayme, D. F. 2018 Geometric decomposition of the conformation tensor in viscoelastic turbulence. J. Fluid Mech. 842, 395-427.

LANG, S. 1999 Fundamentals of Differential Geometry. Springer.

Meneveau, C. \& Katz, J. 2000 Scale-invariance and turbulence models for large-eddy simulation. Annu. Rev. Fluid Mech. 32, 1-32.

Moyers-Gonzalez, M., Owens, R. G. \& FAng, J. 2008 A non-homogeneous constitutive model for human blood. Part 1. Model derivation and steady flow. J. Fluid Mech. 617, 327.

Pan, L., Morozov, A., Wagner, C. \& Arratia, P. E. 2013 Nonlinear elastic instability in channel flows at low Reynolds numbers. Phys. Rev. Lett. 110 (17), 174502.

Samanta, D., Dubief, Y., Holzner, M., SchäFer, C., Morozov, A. N., Wagner, C. \& Hof, B. 2013 Elasto-inertial turbulence. Proc. Natl Acad. Sci. USA 110 (26), 10557-10562.

Shaqfeh, E. S. G. 1996 Purely elastic instabilities in viscometric flows. Annu. Rev. Fluid Mech. 28, 129-185.

Wang, S.-N., Graham, M. D., Hahn, F. J. \& XI, L. 2014 Time-series and extended KarhunenLoève analysis of turbulent drag reduction in polymer solutions. AIChE J. 60 (4), 1460-1475. 